Tema: Metalurgia Secundária

\title{
PREVISÃO DA LIMPIDEZ DO AÇO A PARTIR DA COMPOSIÇÃO QUÍMICA DA ESCÓRIA*
}

\author{
Élida Gonçalves das Neves ${ }^{1}$ \\ Marcus Novaes Motta ${ }^{2}$ \\ José Flávio Viana ${ }^{3}$
}

\section{Resumo}

Nos últimos anos as siderúrgicas têm investido na melhoria de seus processos e produtos buscando à produção de clean steels, onde menor nível inclusionário é almejado. Neste contexto foi objetivado neste trabalho, em um primeiro momento, realizar uma revisão bibliográfica afim de buscar um modelo que pudesse relacionar a composição química da escória com a capacidade da escória de remover de forma eficiente as inclusões, garantindo assim a limpidez de aços. A partir destes dados, buscou-se, como segundo objetivo, avaliar alguns resultados de escórias industriais e compará-los com o proposto pelo modelo. A viscosidade e molhabilidade foram os parâmetros de maior impacto neste estudo, sendo a viscosidade obtida através da termodinâmica computacional com o FactSage e a molhabilidade estimada a partir do modelo de Nakajima.

Palavras-chave: Escória sintética; Inclusão; Molhabilidade; Viscosidade.

\section{FORECAST OF STEEL CLEANING BASAED ON THE CHEMICAL COMPOSITION OF THE SLAG}

\section{Abstract}

In recent years steelmakers have invested in improving their processes and products seeking for the production of clean steels where a lower amounts of inclusions is desired. In this context, it is the objective of this paper, at first, to conduct a literature review in order to find a model that could relate the chemical composition of the slag with its ability to remove the inclusions efficiently, and thus ensuring clean steel. In a second step, based on these data, we evaluated some results of industrial slags and compared them with the proposed model. The most important parameters showing up in this study are viscosity and wettability. The viscosity was obtained using the FactSage software and the wettability was estimated applying the Nakajima model. Keywords: Synthetic slag; Inclusion; Wettability; Viscosity.

1 Engenheira Metalurgista, Engenheira de Processos, Tecnosulfur S/A, Membro da ABM, Sete Lagoas, MG, Brasil.

2 Engenheiro Metalurgista, Coord. Desenvolvimento de Mercado, Tecnosulfur S/A, Membro da ABM, Sete Lagoas, MG, Brasil.

3 Engenheiro Metalurgista, MsC, CQE, CQA, Gerente de Processos, Tecnosulfur S/A, Membro da ABM, Sete Lagoas, MG, Brasil.

* Contribuição técnica ao 45 Seminário de Aciaria - Internacional, 25 a 28 de maio de 2014, Porto Alegre, RS, Brasil. 


\section{INTRODUÇÃO}

No últimos anos as siderúrgicas têm investido na melhoria de seus processos e produtos. Isto se aplica à produção de clean steels, onde limpidez do aço pode ser compreendida como: menores níveis de elementos residuais na composição química do aço ou menor nível inclusionário. Neste contexto, buscou com este trabalho encontrar na literatura um modelo que permitisse relacionar a composição química da escória com a capacidade da mesma em reter inclusões, garantindo assim a limpidez de aços, e comparar os resultados teóricos com resultados obtidos de processos industriais e criar embasamento teórico para desenvolvimento de escórias sintéticas sinterizadas mais efetivas na retenção de inclusões, já que as mesmas já possuem características que favorecem a otimização dos processos [1].

Muitos estudos tem tratado do processo de remoção de inclusões [2]. Teoricamente a remoção de inclusões não metálicas a partir de aço fundido pode ser conseguida por meio de vários mecanismos diferentes. De acordo com a lei de Stokes, devido às diferenças entre as densidades das inclusões não metálicas e do aço, as inclusões flotam até a superfície. A flotação é o mecanismo que poderia conduzir à remoção de inclusões não-metálicos e teoricamente é possível calcular a taxa de inclusão retirada por flotação. Outro mecanismo para remoção de inclusões é o uso de agitação eletromagnética ou por gás inerte

Os mecanismos acima citados podem ser usados para entender o movimento da inclusão no aço desde o fundo da panela até a superfície do aço, entretanto, sem uma escória com propriedades adequadas estas inclusões podem não ser removidas efetivamente.

Inclusões são geralmente removidas por reação com escória. Isto é conseguido principalmente por otimização das condições do processo para promover o contato e reação entre a inclusão e a escória. Para a remoção eficiente, as inclusões devem juntar-se e dissolver-se na fase de escória. Se a interação escória/inclusão é fraca, então as inclusões estarão sujeitas a serem reincorporadas no metal. A força de fixação (reatividade) entre a inclusão e a fase de escória pode ser caracterizada pela molhabilidade da escória nas inclusões.

A remoção da Inclusão pela escória é dividida em quatro etapas na panela [3]. Na Figura 1, uma ilustração esquemática da parte superior esquerda de uma panela é visto. Primeiro (1.), a inclusão é transportado para uma camada limite pela agitação turbulenta da massa fundida, em seguida (2.), a inclusão é transportado para a interface escória/aço. Posteriormente (3.), separação de inclusão para a fase de escória é possível. Finalmente (4.), a dissolução da inclusão na fase de escória pode ocorrer.

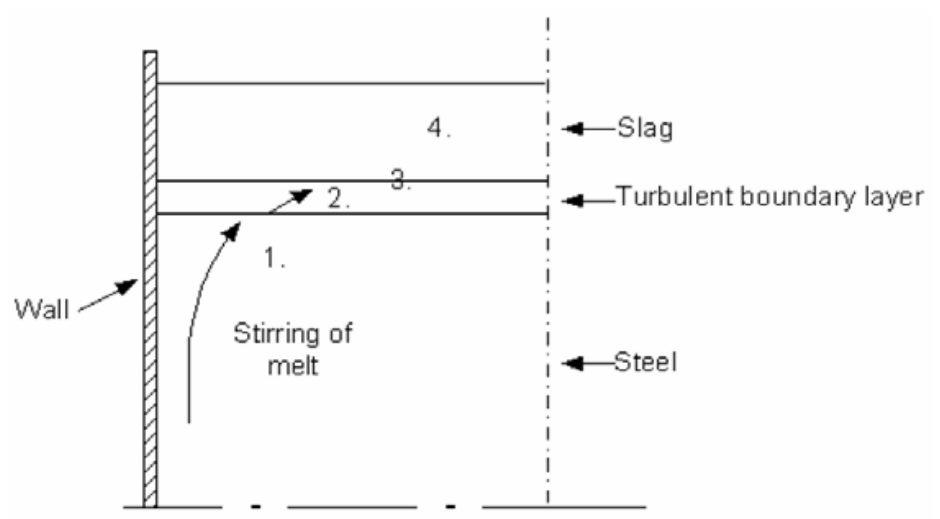

Figura 1. Ilustração esquemática da parte superior esquerda de uma panela com as zonas de remoção inclusão [3].

* Contribuição técnica ao 45 Seminário de Aciaria - Internacional, 25 a 28 de maio de 2014, Porto Alegre, RS, Brasil. 
Neste trabalho serão avaliados apenas a separação na interface inclusão/escória.

\section{SEPARAÇÃO NA INTERFACE INCLUSÃO/ESCÓRIA}

O processo de separação da inclusão não-metálica na interface entre metal e escória é, em grande parte, controlado pelo fenômeno interfacial no sistema metalescória-inclusão. Logo o conhecimento das propriedades interfaciais que governam a separação da inclusão permite o seu controle para alcançar resultados desejados. Durante a permanência do metal liquido na panela, as inclusões podem ser trazidas para a interface metal/escória e, segundo modelo de Nakajima e Okamura utilizado na tese de Wikström [3], pode adotar 3 tipos de comportamento de emersão: 0 modelo passante(pass), o qual é o mais favorável, onde a inclusão está completamente separada do metal, o modelo de permanência (remain) onde a inclusão permanece na interface não permitindo ser totalmente transferida para a escória, e o modelo oscilante (oscillate), onde a inclusão oscila até alcançar um ponto de equilíbrio abaixo da interface. Nos dois últimos casos, a inclusão pode retornar ao banho.

Segundo Strand et al. [4], os parâmetros de maior influência na transferência da inclusão para a escória são a viscosidade da escória e a tensão interfacial. As tensões interfaciais podem ser consideradas como forças e um equilíbrio de força no ponto de contato leva a equação de Youngs, relacionando assim a tensão interfacial com o ângulo de contato entre a inclusão, o metal e a escória (Equação 1), o que determina a molhabilidade ( $\cos \theta \mathrm{IMS})$ da inclusão.

$$
\cos \theta_{I M S}=\frac{\sigma_{I M}-\sigma_{I S}}{\sigma_{M S}}
$$

A molhabilidade da inclusão pelo sistema ocorre quando $\cos \theta \mathrm{IMS}>0$, e se for menor que zero, $\cos \theta \mathrm{IMS}<0$, a molhabilidade não ocorre.

A Figura 2 mostra uma comparação do comportamento da inclusão em função da molhabilidade e viscosidade da escória. A Figura 3 mostra a variação de posição da inclusão na interface quando a viscosidade e tensão interfacial entre metal e escória são mantidas constantes e há um aumento na molhabilidade. Pode-se ver que, com o aumento da molhabilidade, mais a inclusão emerge na escória, evitando assim o seu retorno ao banho.

\footnotetext{
* Contribuição técnica ao 450 Seminário de Aciaria - Internacional, 25 a 28 de maio de 2014, Porto Alegre, RS, Brasil.
} 

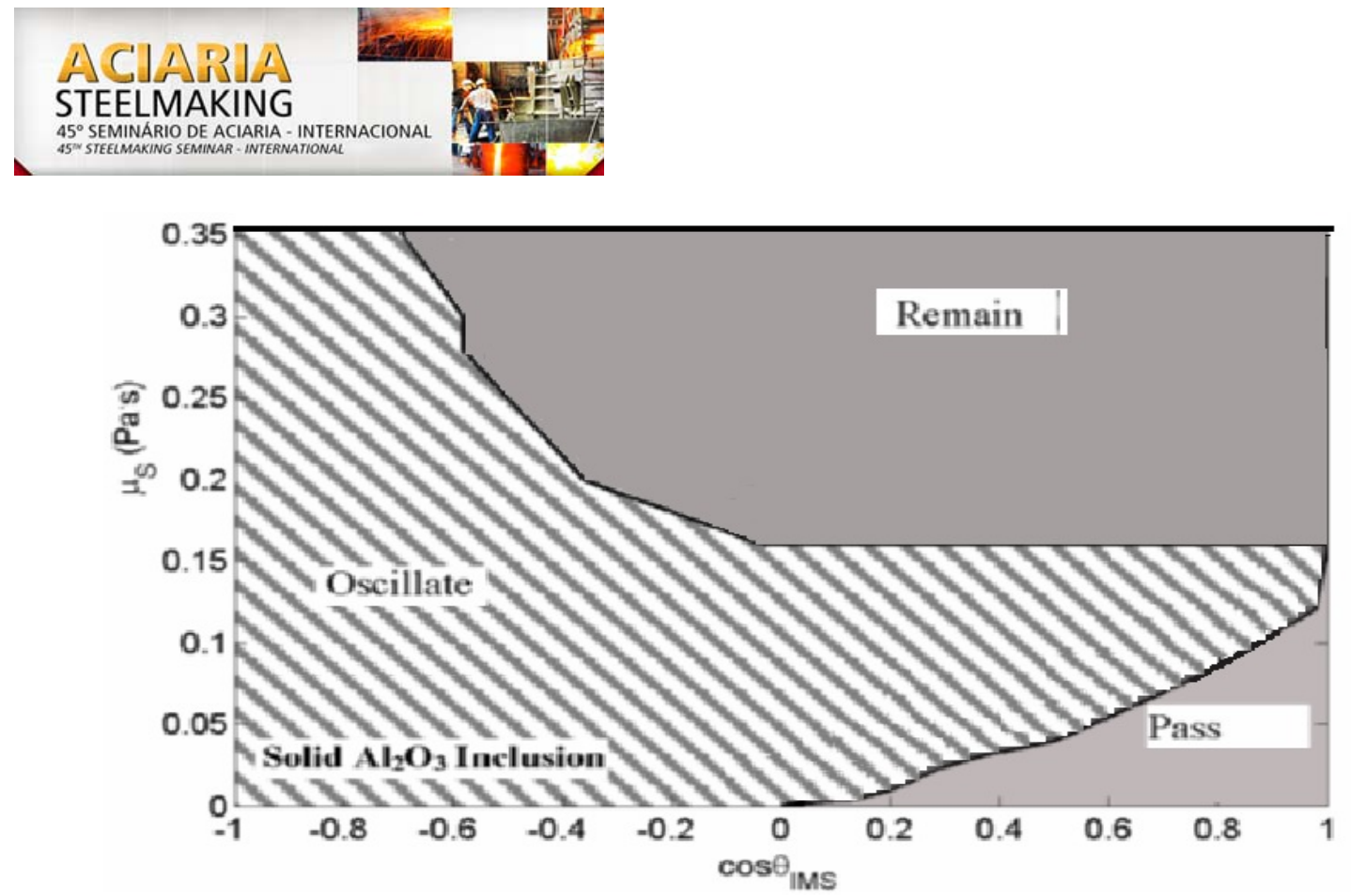

Figura 2. Comparação do comportamento da inclusão em função da molhabilidade e viscosidade da escória de uma inclusão solida (Al2O3) menor que 100 $\mu \mathrm{m}$ [3].

a)

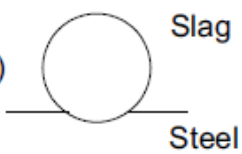

b)

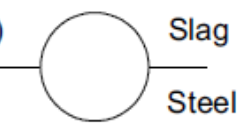

c)

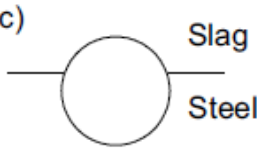

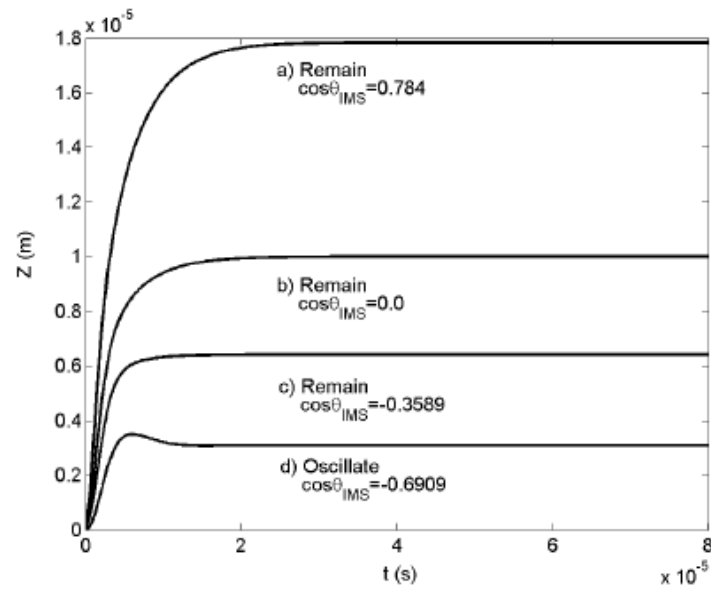

Figura 3. Variação da posição da escória no banho, quando altera a molhabilidade, mas é mantido constante a viscosidade da escória e a tensão interfacial entre o metal e a escória [3].

Strand através de testes com escórias em condição industrial concluiu que a molhabilidade deve ser elevada e o valor da viscosidade baixo para se obter inclusões completamente emergidas na escória e logo uma ótima transferência de inclusão, entretanto deve-se ter cuidado com viscosidades de escórias muito baixas para se evitar arraste de escória para dentro do banho resultando em mais inclusões no aço.

Choi et al. [5] também investigou o comportamento da molhabilidade do substrato de alumina por diversas escórias $\mathrm{CaO}-\mathrm{Al} 2 \mathrm{O} 3-\mathrm{SiO} 2$ há $1600^{\circ} \mathrm{C}$.

Na Figura 4 tem-se o ângulo de contato entre a escória e a inclusão de alumina. Quanto menor o ângulo de contato entre escória/inclusão maior será a molhabilidade da inclusão pela escória e, portanto maior chance de remoção da inclusão do banho metálico ser incorporado pela escória. Podemos ver que na região de baixo teor de $\mathrm{SiO} 2$, a escória com alto teor de $\mathrm{CaO}$ exibe um menor ângulo, o que favorece a molhabilidade com a alumina. Para uma escória com uma dada basicidade binária $\mathrm{CaO} / \mathrm{SiO} 2$, um aumento no teor de $\mathrm{Al} 2 \mathrm{O} 3$ leva a um aumento no ângulo de contato, isto é, diminui a molhabilidade.

* Contribuição técnica ao 45 Seminário de Aciaria - Internacional, 25 a 28 de maio de 2014, Porto Alegre, RS, Brasil. 

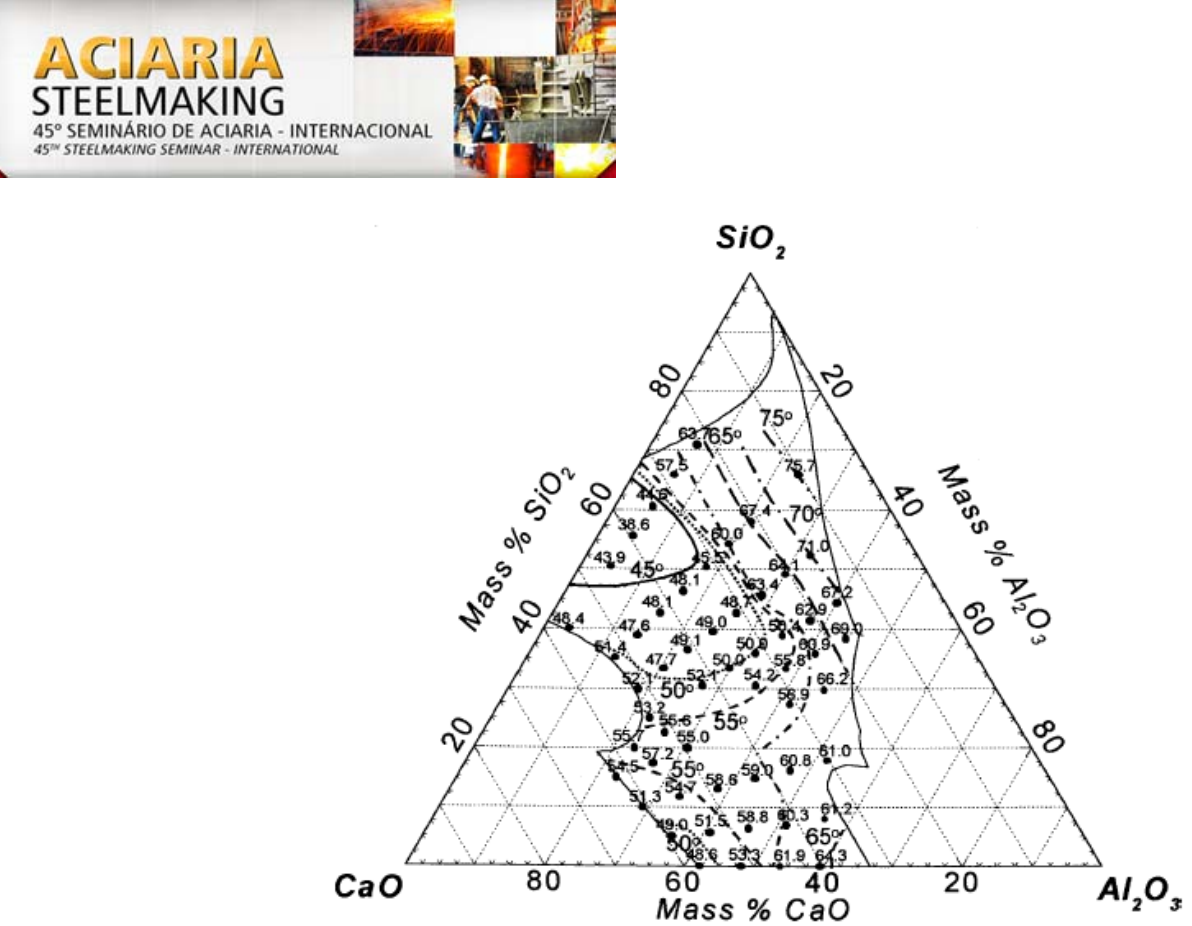

Figura 4. Ângulo de contato entre a escória (CaO-SiO2-Al2O3) e a alumina à $1873 \mathrm{~K}$ [5].

A Figura 5 mostra a tensão interfacial do sistema CaO-SiO2-Al2O3/ inclusão de $\mathrm{Al}$ 2O3. A tensão interfacial está diretamente relacionada ao ângulo de contato, logo quanto menor a tensão interfacial, menor o ângulo de contato e, portanto, maior molhabilidade da inclusão pela escória.

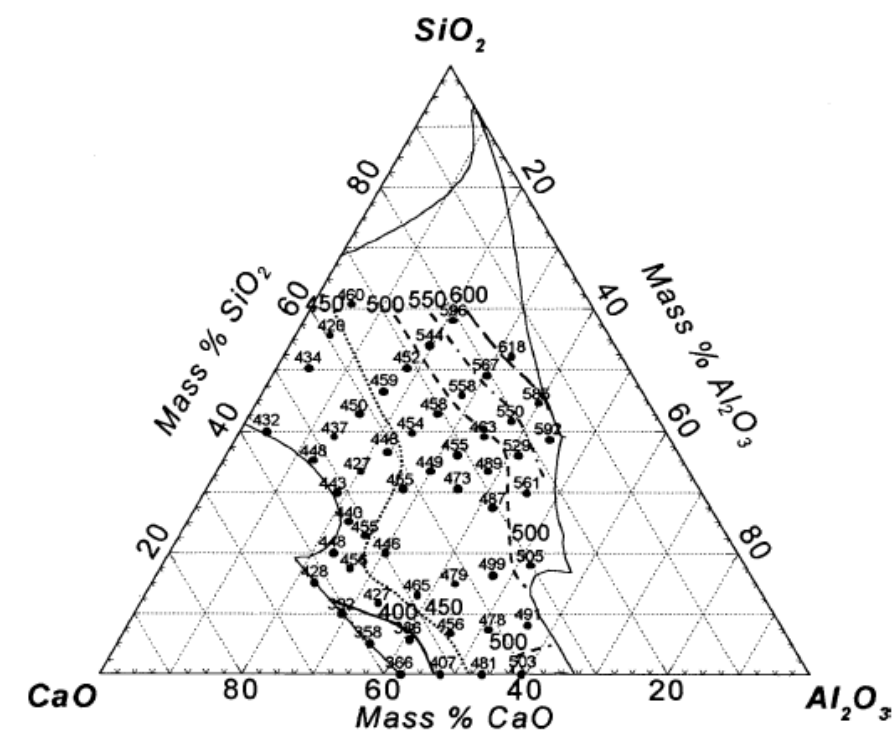

Figura 5. Tensão interfacial (x 10-3 Nm-1) entre a escória (CaO-SiO2-Al2O3) e a inclusão de Al2O3 [5].

\section{PARÂMETROS DO MODELO MATEMÁTICO}

De acordo com a literatura pesquisada este estudo só lida com a separação de uma inclusão localizados apenas na interface aço-escórias. Assim, não considera a dissolução da inclusão na escória, nem a criação de novas inclusões devido à reoxidação.

Como mencionado anteriormente este modelo propõe 3 tipos de comportamento da escória (passante, permanência e oscilante), sendo a viscosidade (visc) da escória um dos parâmetros importante neste modelo e foi obtido com uso da matemática computacional (FactSage). Outro parâmetro importante foi a tensão interfacial entre

* Contribuição técnica ao 450 Seminário de Aciaria - Internacional, 25 a 28 de maio de 2014, Porto Alegre, RS, Brasil. 
escória-inclusão ( $\sigma S I)$, inclusão-metal $(\sigma \mathrm{IM})$ e metal-escória $(\sigma \mathrm{MS})$, e a partir desses valores, foi calculada a molhabilidade ( $\cos \theta \mathrm{IMS}$ ). Devido à dificuldade para encontrar dados experimentais para cálculo da tensão interfacial, a mesma foi estimada. As equações para tensão interfacial, tensão superficial do metal $(\sigma \mathrm{M})$, da escória $(\sigma S)$ e da inclusão $(\sigma \mathrm{l})$, coeficiente de interação metal/escória $(\phi M S)$ e escória/inclusão $(\phi S I)$, tensão interfacial entre inclusão/metal $(\sigma \mathrm{IM})$, metal/escória $(\sigma \mathrm{MS})$ e escória/inclusão ( $\sigma \mathrm{SI})$ podem ser encontradas nas referências 6,7 e 8 [6-8].

Para o estudo foram utilizados dados de escórias de final de processo de aciaria e resultados de limpidez de aço já publicados [9] e foram considerados para cálculos como sendo inclusão solida de $\mathrm{Al} 2 \mathrm{O} 3$ para o processo de rotina e inclusão com relação $\mathrm{Ca} / \mathrm{Al}>0,14$ [10] para o processo alterado.

\section{RESULTADOS}

A Tabela 1 apresenta os dados da composição de escória utilizados nos cálculos.

Tabela 1. Teores Médios de $\mathrm{CaO}, \mathrm{Al} 2 \mathrm{O} 3$ e FeO das escórias após tratamento no forno panela para os dois processos em questão [9]

\begin{tabular}{|l|cc|c|}
\cline { 2 - 4 } \multicolumn{1}{c|}{} & $\mathrm{CaO}$ & $\mathrm{Al}_{2} \mathrm{O}_{3}$ & $\mathrm{FeO}$ \\
\hline Processo normal & 41,28 & 29,12 & 4,39 \\
\hline Após alteração processo & 57,59 & 25,80 & 1,39 \\
\hline
\end{tabular}

Os dados de viscosidade obtidos com o FACTSAGE e os valores estimados para cálculo de tensões podem ser visto na Tabela 2.

Tabela 2. Dados obtidos através do FactSage e estimados para utilização do modelo

\begin{tabular}{|c|c|c|c|c|c|c|c|c|c|c|c|c|}
\hline & visc(Pa.s) & $\sigma \mathrm{m} / \mathrm{N} / \mathrm{m}$ & $\sigma s(\mathrm{~N} / \mathrm{m}$ & $\sigma s(\mathrm{~N} / \mathrm{m})$ & $\sigma \mid(\mathrm{N} / \mathrm{m}$ & $\sigma \mathrm{\sigma l} / \mathrm{N} / \mathrm{m}$ & $\phi M S$ & $\phi S I$ & $\sigma \mid \mathrm{M}$ & $\sigma \mathrm{MS}$ & $\sigma \mathrm{SI}$ & $\cos \theta$ IMS \\
\hline Processo normal & 0,122 & 1,649 & 0,609 & 0,639 & 0,646 & 0,676 & 0,376 & 0,651 & 1,380 & 1,515 & 0,460 & 0,607 \\
\hline Após alteração processo & 0,119 & 1,649 & 0,608 & 0,638 & 0,638 & 0,668 & 0,448 & 0,736 & 1,378 & 1,368 & 0,344 & 0,755 \\
\hline
\end{tabular}

Com os dados acima foi possível plotar sobre o gráfico proposto pela literatura (Figura 6) e determinar a condição das escórias utilizadas no processo industrial e com isso comparar com os resultados de inclusões apresentados na literatura (Figura 7) para validar o modelo proposto. Como não há grandes alterações entre a representação gráfica para inclusão de $\mathrm{Al} 2 \mathrm{O} 3$ e $\mathrm{CaO}-\mathrm{Al} 2 \mathrm{O} 3$, para efeito de simplificação os dados foram plotados sobre a referência de inclusão de alumina.

\footnotetext{
* Contribuição técnica ao 45 Seminário de Aciaria - Internacional, 25 a 28 de maio de 2014, Porto Alegre, RS, Brasil.
} 

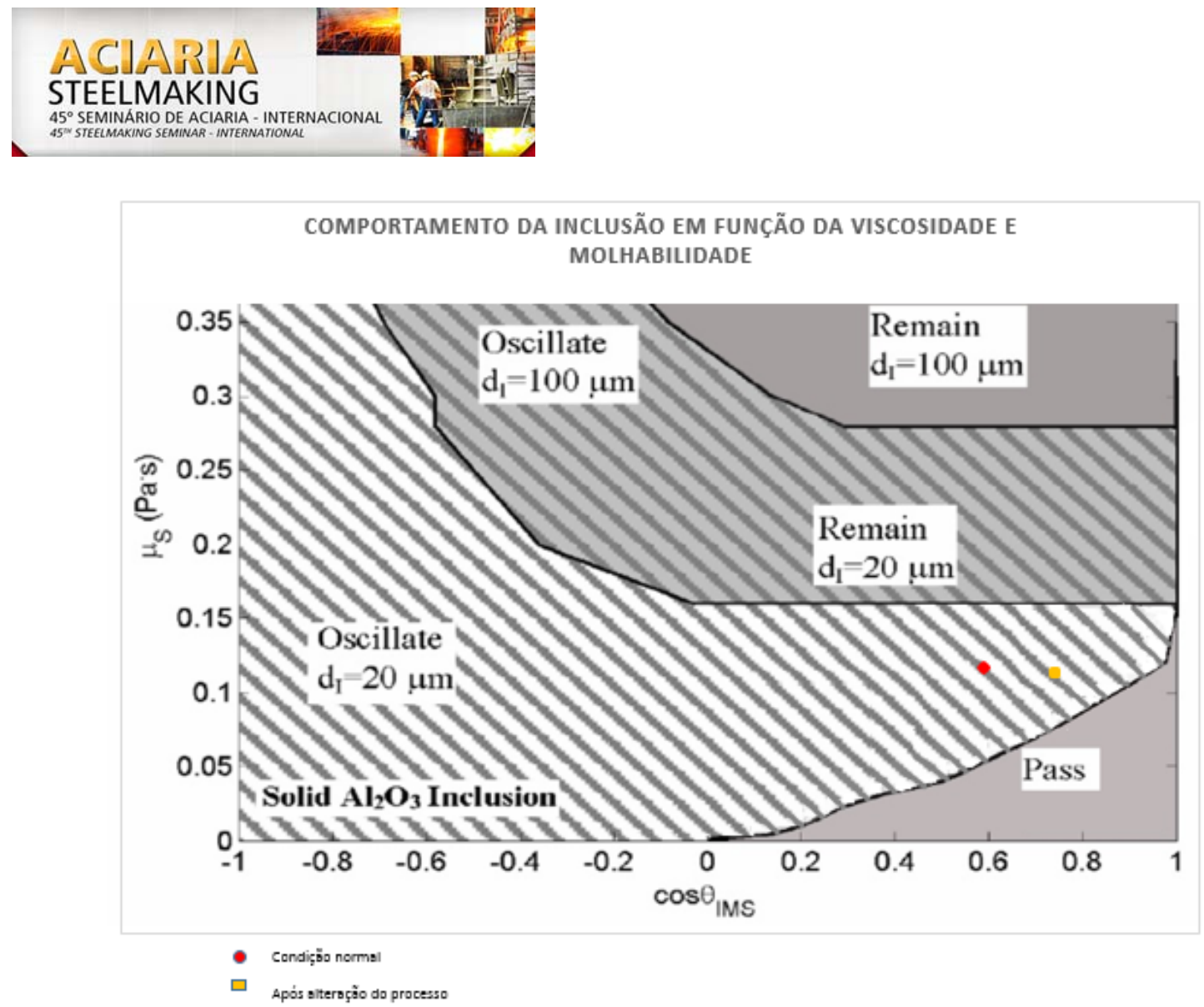

Figura 6. Relação estimada entre viscosidade da escória e molhabilidade para condição industrial.

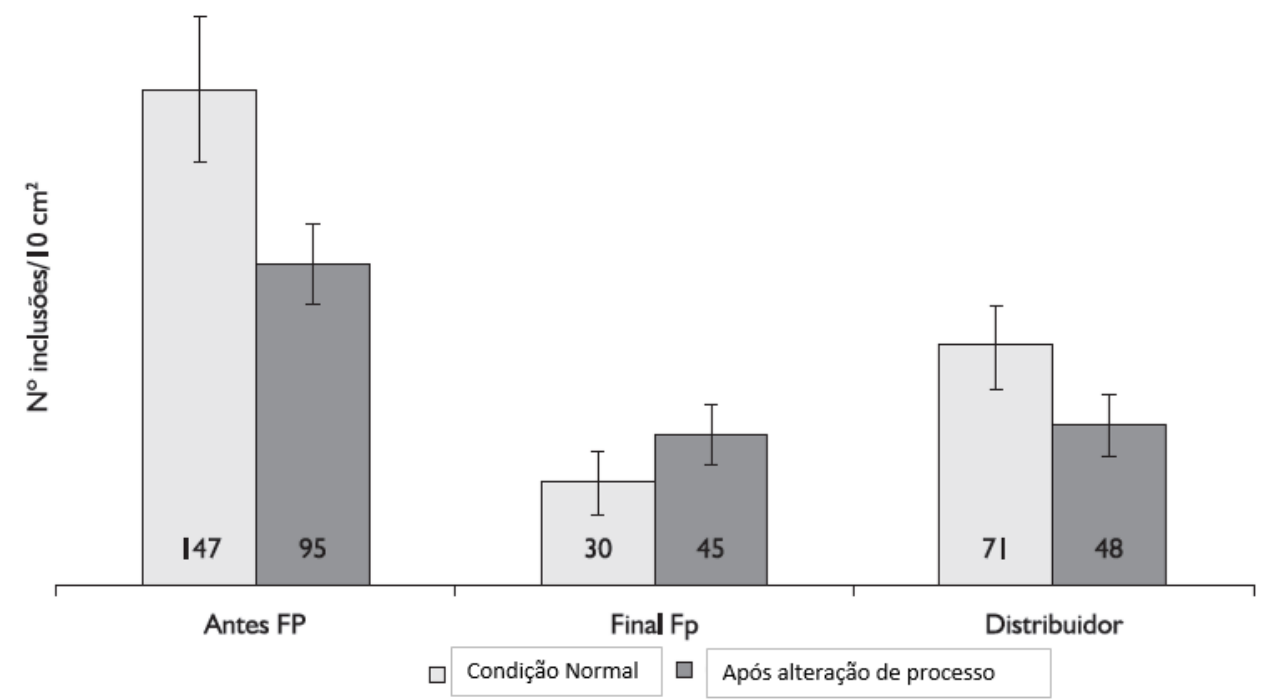

Figura 7. Variação do número de inclusões de alumina ao longo do processamento do aço (antes e após o tratamento no Forno Panela e aos 50\% do lingotamento no Distribuidor) [9].

\section{DISCUSSÃO}

O modelo em questão, como já mencionado, lida com a separação de uma inclusão localizados apenas na interface aço-escórias. Ele relaciona o efeito da viscosidade da escória e molhabilidade sobre a capacidade da escória em melhorar a limpidez do aço, ou seja, escórias com menor viscosidade e maior valor de molhabilidade terá maior capacidade de reduzir o nível de inclusões no aço. Segundo o modelo, a relação viscosidade-molhabilidade propõem que não há retorno da inclusão para o banho, apenas no caso dos resultados que estiverem no campo passante (pass), nos demais caso poderá ocorrer o retorno da inclusão para o banho.

\footnotetext{
* Contribuição técnica ao $45^{\circ}$ Seminário de Aciaria - Internacional, 25 a 28 de maio de 2014, Porto Alegre, RS, Brasil.
} 
Os resultados obtidos mostram que após a alteração do processo, ocorrida no forno panela (FP), a escória final obtida teve o mesmo comportamento que a condição de rotina, chamada aqui de normal. Ambas encontram-se no campo oscilante (oscillate), o qual pode ocorrer o retorno da inclusão para o banho. A Figura 7 mostra que mesmo após a alteração de processo houve presença de inclusão no final de processo, entretanto o resultado da condição após alteração do processo mostrou-se melhor, mais estável que o processo normal, o que pode ser explicado, desconsiderando os ruídos do processo, de acordo com o modelo proposto, pela menor viscosidade e maior molhabilidade da inclusão pela escória melhorando assim a captação de inclusões.

\section{CONSIDERAÇÕES FINAIS}

O trabalho teve como objetivo mostrar a viabilidade do modelo para um processo industrial, mostrando-se a primeira vista efetivo para o entendimento do comportamento das inclusões no banho durante o processo, permitindo agora um estudo mais aprofundado. Busca-se agora um acompanhamento maior de um processo industrial para relacionar os parâmetros do modelo com os resultados de inclusão no processo final considerando os diversos ruídos existentes no processo e a partir daí desenvolver escórias sintéticas sinterizadas que possam, além dos diversos benefícios que elas já possuem [11], serem projetadas com a finalidade de redução efetiva de inclusões.

\section{REFERÊNCIAS}

1 Viana JF, Santos, MA, Lourenço D, Farage SD, Oliveira JR. Estudo das características de fusão das escórias sintéticas sinterizadas. In: $43^{\circ}$ Seminário de Aciaria -

Internacional; 2012; Belo Horizonte, Brasil. São Paulo: ABM; 2012.

2 Riyahimalayeri K. Slag, steel, ladle and non-metallic inclusions equilibria in an ASEASKF ladle furnace. Stockholm: KTH Royal Institute of Technology; 2012.

3 Wikström J. A mathematical and experimental study of inclusion behavior at a steel-slag interface. Stockholm: KTH Royal Institute of Technology; 2007.

4 Strandh J, Nakajima K, Eriksson R, Jönsson P. Solid inclusion transfer at a steel-slag interface with focus on tundish conditions. ISIJ International. 2005;45(11):1597-606.

5 Choi J, Lee H. Wetting of solid Al2O3 with molten $\mathrm{CaO}-\mathrm{Al} 2 \mathrm{O} 3-\mathrm{SiO} 2$. ISIJ International. 2003;43(9):1348-55.

6 Nakajima K. Estimation of surface tension for multicomponent silicate melts. Tetsu-toHagane. 1994;80(8):599-604.

7 Nakajima K. Estimation of interfacial tensions between phases in the molten iron-slaginclusion (alumina) system. Tetsu-to-Hagane. 1994;80(5):383-388.

8 Mills K. The estimation of slag properties. London: Department of Materials, Imperial College; 2011.

9 Filho ACPC, Souza CA, Junior JDGA, Freitas PA, Rocha SH, Menezes GA, et al. Carbureto de cálcio: uma alternativa eficiente ao uso de alumínio. Tecnol. Mater. Miner. 2013;10(1):28-34.

10 Magalhães HLG. Melhoria da limpidez do aço SAE 1045 desoxidado ao alumínio com aplicação na indústria automobilística, utilizando termodinâmica computacional. Ouro Preto: UFOP; 2010.

11 Viana JF, Oliveira JR, Gillo FF, Sampaio RA, Motta MN. Estudo da dessulfuração de aço com utilização de escória sintética sinterizada. In: $44^{\circ}$ Seminário de Aciaria Internacional; 2013; Araxá, Brasil. São Paulo: ABM; 2013.

\footnotetext{
* Contribuição técnica ao 45 Seminário de Aciaria - Internacional, 25 a 28 de maio de 2014, Porto Alegre, RS, Brasil.
} 